\title{
ADAPTAÇÃO TRANSCULTURAL DA ESCALA JEFFERSON DE ATITUDES RELACIONADAS À COLABORAÇÃO INTERPROFISSIONAL
}

\section{ARTIGO ORIGINAL}

ABED, Marcelo Musa ${ }^{1}$, PEREIRA, Edna Regina Silva², GROSSEMAN, Suely ${ }^{3}$

ABED, Marcelo Musa. PEREIRA, Edna Regina Silva. GROSSEMAN, Suely. Adaptação transcultural da Escala Jefferson de atitudes relacionadas à colaboração interprofissional. Revista Científica Multidisciplinar Núcleo do Conhecimento. Ano. 06, Ed. 10, Vol. 06, pp. 22-44. Outubro 2021. ISSN: 2448-0959, Link de acesso: https://www.nucleodoconhecimento.com.br/saude/escala-jefferson, DOI: 10.32749/nucleodoconhecimento.com.br/saude/escala-jefferson

\section{RESUMO}

A colaboração interprofissional está no foco da transformação do processo de trabalho entre profissionais de diversas categorias demonstrando que uma formação interprofissional pode garantir a prática cooperativa nos serviços de saúde. $A$ Jefferson Scale of Attitudes Toward Interprofessional Collaboration permite avaliar a colaboração interprofissional. Sua versão em português, a Escala Jefferson de Atitudes Relacionadas à Colaboração Interprofissional, seguiu os passos para adaptação, finalizando com a avaliação por comitê de especialistas quanto à equivalência cultural e conceitual em relação ao seu objetivo e aplicação. No presente artigo, a escala foi aplicada em uma amostra de 128 profissionais da

\footnotetext{
${ }^{1}$ Mestrado em Ensino na Saúde (UFG), pós-graduação em Acupuntura (IMES) e em Saúde Pública com ênfase em Saúde da Família (CGESP), Graduação em Enfermagem (UFU). ORCID: http://orcid.org/0000-0002-25816941

${ }^{2}$ Orientadora. Pós-doutorado em Educação Médica (UNICAMP), Doutorado em Medicina Nefrologia (USP), Residência Médica Em Nefrologia (HSPE-SP), Graduação em Medicina (UFMA). ORCID: https://orcid.org/00000001-7177-3893

${ }^{3}$ Coorientadora. Pós-Doutorado em Drexel University College of Medicine (DUCOM, Estados Unidos), Doutorado em Engenharia de Produção (UFSC), Mestrado em Mother and Child Health (University of London, UL, Inglaterra), Especialização em Educação para as Pofissões de Saúde (UFC), Residência Médica Em Pediatria (UFRJ), Graduação em Medicina (UFRJ). ORCID: http://orcid.org/0000-0002-2039-9827
}

RC: 99337

Disponível em: https://www.nucleodoconhecimento.com.br/saude/escala-jefferson 
Atenção Básica. A validação de conteúdo foi obtida por meio do Índice de Validação de Conteúdo (IVC) obtendo o resultado de 0,99, demonstrando que a escala é capaz de medir aquilo que se propõe. Conclui-se que a adaptação transcultural e validação foram adequadamente realizadas para a língua portuguesa brasileira. O valor Alfa de Cronbach calculado para a escala foi de 0,71, demonstrando uma boa confiabilidade interna da escala.

Palavras-chave: Estudos de Validação, Equipe de Assistência ao Paciente, Escalas, Questionários, Atenção Primária à Saúde.

\section{INTRODUÇÃO}

A educação interprofissional permite uma prática colaborativa eficaz, capaz de aperfeiçoar serviços de saúde, fortalecer as redes e melhorar os resultados de saúde. É possível perceber níveis mais elevados de satisfação dos pacientes, bem como melhor aceitação do atendimento e melhores resultados de saúde, após receberem atenção de uma equipe colaborativa. (WHO, 2010).

No Brasil, a prática colaborativa ( $P C$ ) é preconizada na Atenção Básica ( $A B)$, segundo a Política Nacional de Atenção Básica (PNAB), que descreve a $A B$ como principal porta de entrada do SUS e centro de comunicação com toda a Rede de Atenção à Saúde (RAS). (BRASIL, 2017).

Desde a sua criação, a Estratégia Saúde da Família (ESF) atua através de equipe multiprofissional com população adstrita e referenciada sob sua responsabilidade. Essas equipes possuem composição mínima formada por enfermeiro, técnico ou auxiliar de enfermagem, médico generalista e Agente Comunitário de Saúde (ACS). Uma atribuição comumente desejada dos profissionais é a realização do trabalho interprofissional e em equipe. (BRASIL, 2017)

Para Silva et al. (2015) e Wiecha e Pollard (2004) a prática interprofissional nos serviços de saúde tem sido cada vez mais valorizada, devido às significativas mudanças no perfil demográfico e epidemiológico da população, com aumento da 
expectativa de vida dos indivíduos e consequente aumento das doenças crônicodegenerativas exigindo segundo Fortuna et al. (2005) uma abordagem interdisciplinar para a atenção integral à saúde.

Peduzzi et al (2011) afirma que o trabalho em equipe para ser desenvolvido, é necessário um conjunto de ações como a construção e consolidação de espaços de troca entre os profissionais, estímulo aos vínculos profissional-usuário e usuárioserviço e à autonomia das equipes. A competência de cada profissional, isoladamente, não é capaz de suprir as necessidades de saúde de um indivíduo, sendo, portanto, necessária uma flexibilidade nos limites das competências para proporcionar uma ação integral. (AGUILAR DA SILVA, SCAPIN e BATISTA, 2011)

Entretanto, ainda há um caminho a ser percorrido para alcançar um trabalho em equipe colaborativo nos serviços de saúde, quer sejam públicos ou privados. Exemplo disso é abordado por Trad (2005) em que a ausência de responsabilidade coletiva do trabalho e baixo grau de interação entre as categorias profissionais em equipes da ESF que apresentam hierarquização entre as profissões, comunicação superficial e limitada a aspectos técnicos, que comprometem a atuação da equipe. Logo, a estruturação de equipes multiprofissionais na ESF não é garantia de mudança do modelo de atendimento centralizado no profissional médico. (DE SOUZA ARAÚJO e DE MEDEIROS ROCHA, 2007).

Apesar da importância do trabalho em equipe, a pesquisa empírica sobre as relações de colaboração entre diversos profissionais da saúde não tem recebido atenção suficiente. Como primeiro passo para comparação das relações entre os profissionais de saúde, é necessária a utilização de instrumentos de pesquisa de caráter multidimensional e psicometricamente validados que possam avaliar essas relações de colaboração interprofissional. (HOJAT et al., 2002).

A utilização da educação interprofissional e a PC podem desempenhar um papel importante na redução de muitos desafios enfrentados pelos sistemas de saúde no mundo. Profissionais de saúde que trabalham em equipe com PC podem aumentar a capacidade dos serviços de responderem a demandas de saúde, estando mais 
preparados para enfretamento de epidemias e pandemias que são situações que geram solicitações inesperadas e intensas ao sistema de saúde. (OMS, 2010).

É nesse cenário que a melhoria da qualidade da atenção à saúde exige uma formação profissional com conhecimentos específicos, domínio de tecnologias que qualifiquem a atenção individual e coletiva e capacidade de atuação em equipe multiprofissional. (BRASIL, 2017)

Em 2001 Ministério da Educação publicou as primeiras Diretrizes Curriculares Nacionais (DCN) dos Cursos de Graduação em saúde, nas quais trazem como perfil do egresso, habilidades e competências para atuar no trabalho em equipe. No curso de medicina a habilidade específica: de assumir o compromisso ético, humanístico e social com o trabalho multiprofissional em saúde; e no curso de enfermagem de atuar em equipe multiprofissional. (BRASIL, 2001a, 2001b).

Recentemente, em 2014, as DCN para a graduação em medicina foram atualizadas para uma atuação do médico em equipe multiprofissional para a aprendizagem interprofissional. Esse avanço observado permite intensificar a colaboração entre estes profissionais e a equipe no atendimento integral de saúde. (BRASIL, 2014).

Apesar de integralidade ser um eixo transversal nas competências de todos os profissionais da saúde, a formação acadêmica dissociada de uma educação multiprofissional não é capaz de preparar o egresso para o trabalho em equipe. (CECCIM e FEUERWERKER, 2004).

É importante ressaltar que o trabalho em equipe não tem na sua origem apenas o caráter de racionalização da assistência médica, no sentido de garantir a melhor relação custo-benefício do trabalho médico e ampliar o acesso e a cobertura da população atendida, mas também responde à necessidade de integração das disciplinas e das profissões, entendida como imprescindível para o desenvolvimento das práticas de saúde a partir da nova concepção biopsicossocial do processo saúde-doença. (PEDUZZI e CIAMPONE, 2005). 
Iribarry (2003) relata que a realidade de trabalho presente na atualidade teve sua origem na disciplinaridade, processo que surgiu no século XVII devido à fragmentação da ciência, que determinou a divisão do conhecimento em disciplinas. Já no início do século XX, Lampert (2004) diz que o ensino e a prática médica enfatizavam a dimensão biológica, a doença e a medicina curativa. Esse modelo teve início em 1910 e segundo Cooke et al (2006) ficou conhecido como paradigma flexneriano. Na tentativa de unificar essa fragmentação disciplinar, surge a estratégia de integração entre as disciplinas, o que futuramente vai evoluir através dos conceitos de multi, pluri, inter e transdisciplinaridade, cada uma com maior ou menor grau de conexão entre as disciplinas. (ALONSO, 2005).

A diferença básica entre os conceitos de multidisciplinaridade e interdisciplinaridade, é que, no primeiro, as pessoas desenvolvem seu trabalho sem haver uma integração de saberes, com a atuação profissional ocorrendo de forma distinta e independente. Já na segunda, há uma troca de saberes, uma escuta coletiva, gerando uma nova configuração interna sobre aquele conhecimento e nisso se reproduz a atitude interdisciplinar. (SOMMERMAN, 2006).

Para Lemos; Reineiros e de Morais (2006) a interdisciplinaridade pode ser entendida como uma proposta de integração disciplinar e de mudança nas práticas para abordagem ampliada das necessidades de saúde, mas representa uma experiência desafiadora que requer mudanças dos modelos de atenção reforça Peduzzi (2013) e inclui, no âmbito do trabalho em equipe, a tolerância, o respeito, e a superação das verticalizações de poder para uma situação de maior equilíbrio e interdependência das diversas áreas frente competição e fragmentação. (SOUZA et al., 2016)

Sendo a educação e prática interprofissional (EIP e PIP) as formas de se alcançar a interdisciplinaridade e a PC. Segundo a Organização Mundial de Saúde (2010), ocorre educação interprofissional quando estudantes de duas ou mais profissões aprendem sobre os outros, com os outros e entre si para possibilitar a efetiva colaboração e melhorar os resultados na saúde através da PC. Considera-se que essa prática capaz de fechar as lacunas das disparidades de saúde, tendo a colaboração interprofissional como base para atuação conjunta de prestadores de 
cuidados de saúde, na busca de atender às necessidades do paciente. (VANDERBILT; DAIL e JABERI, 2015).

Camara et al. (2016) reforça que cenário brasileiro apresenta uma formação em saúde majoritariamente uniprofissional em que as iniciativas de educação interprofissional surgem como novo paradigma e tem despontado na graduação, abrindo uma nova perspectiva de formação de profissionais de saúde. Vale citar Peduzzi (2013) que relata algumas ações multiprofissionais que tem ocorrido durante o processo de graduação e pós-graduação latu sensu, como a Residência Multiprofissional em Saúde, ou nas atividades optativas extracurriculares, por exemplo, no Programa de Educação pelo Trabalho para Saúde (PET-Saúde). Essas abordagens visam mudar a concepção de trabalho em equipe na saúde no Brasil, onde ainda há predomínio da noção de equipe multidisciplinar, em que diferentes profissões compartilham o mesmo espaço físico e a mesma clientela, não assegurando, necessariamente, uma integralidade da atenção à saúde. (PEDUZZI e CIAMPONE, 2005).

Nesse sentido, Hojat e Herman (1985) projetam a avaliação da colaboração entre profissionais de saúde como uma necessidade e os estudos devem ser realizados através de ferramentas multidimensionais, que sejam capazes de medir as atitudes em relação à colaboração profissional, um exemplo é a escala desenvolvida por eles para colaboração entre médico-enfermeiro, que avalia diferentes aspectos das relações entre esses dois profissionais. Nos últimos 30 anos diversas escalas surgiram de forma a avaliar diferentes aspectos da colaboração entre profissões distintas.

Pesquisa realizada pelo Canadian Interprofessional Health Collaborative (CIHC) em 2012, construiu-se um inventário de instrumentos quantitativos de medição de Educação Interprofissional e Prática Colaborativa. A revisão identificou 128 ferramentas quantitativas relevantes para a educação interprofissional ou prática colaborativa. Elas foram separadas por nível da seguinte forma: 64 instrumentos relacionados à atitude, 20 de conhecimentos e habilidades, 34 para comportamento, 6 relacionados ao nível organizacional, 8 à satisfação do paciente e os 14 restantes 
para satisfação do gestor. (CIHC - CANADIAN INTERPROFESSIONAL HEALTH COLLABORATIVE, 2012).

Dentre os 64 instrumentos relacionados à atitude, encontra-se a Jefferson Scale of Attitudes toward Physician-Nurse Collaboration. Desenvolvida por Hojat et. al. em 1999, que avalia a interação médico-enfermeiro (a). A ferramenta possui 20 itens com escalas de "Likert" de quatro pontos e já foi traduzida e validada na Itália, México e Israel. (HOJAT et al., 2002).

Diante dos desafios enfrentados na $A B$ e na ESF e pela necessidade de avançar na difusão e organização de práticas colaborativas nos diferentes ambientes de trabalho de profissionais e equipes de saúde, buscou-se suprir a necessidade de um instrumento que avalie os diferentes níveis de relacionamento e colaboração entre as diferentes profissões da saúde. Nesse sentido, optou-se pela Jefferson Scale of attitudes toward interprofessional collaboration (JeffSATIC), devido a seu reconhecido valor e amplo escopo para avaliação interprofissional. (HOJAT et al., 2015).

O objetivo deste artigo é o de apresentar as etapas do processo de validação para o português da escala JeffSATIC, cuja construção da versão é apresentada sua adaptação transcultural e tradução final com o título de Escala Jefferson de Atitudes Relacionadas à Colaboração Interprofissional (EJARCI).

A JeffSATIC é um instrumento que permite avaliar como é percebido o trabalho interprofissional desenvolvido entre profissionais de saúde. Possui 20 itens que devem ser respondidos utilizando uma escala de concordância ou discordância tipo "Likert" com sete níveis, sendo o menor nível representado por um (1), equivalente a discordo completamente e o maior nível representado por sete (7), equivalente a concordo completamente. (HOJAT, WARD, et al., 2015). 


\section{MÉTODO}

A Adaptação Transcultural foi realizada com base no guia de referência para estudos de adaptação transcultural de instrumentos de saúde a adaptação transcultural do instrumento apresentada por Beaton et al. (2000) e deve ser realizada em cinco estágios: tradução, síntese, retro tradução, comitê de especialistas e pré-teste. A Figura 1 descreve os estágios da adaptação transcultural.

Figura 1: Fluxograma representativo dos estágios da adaptação transcultural.

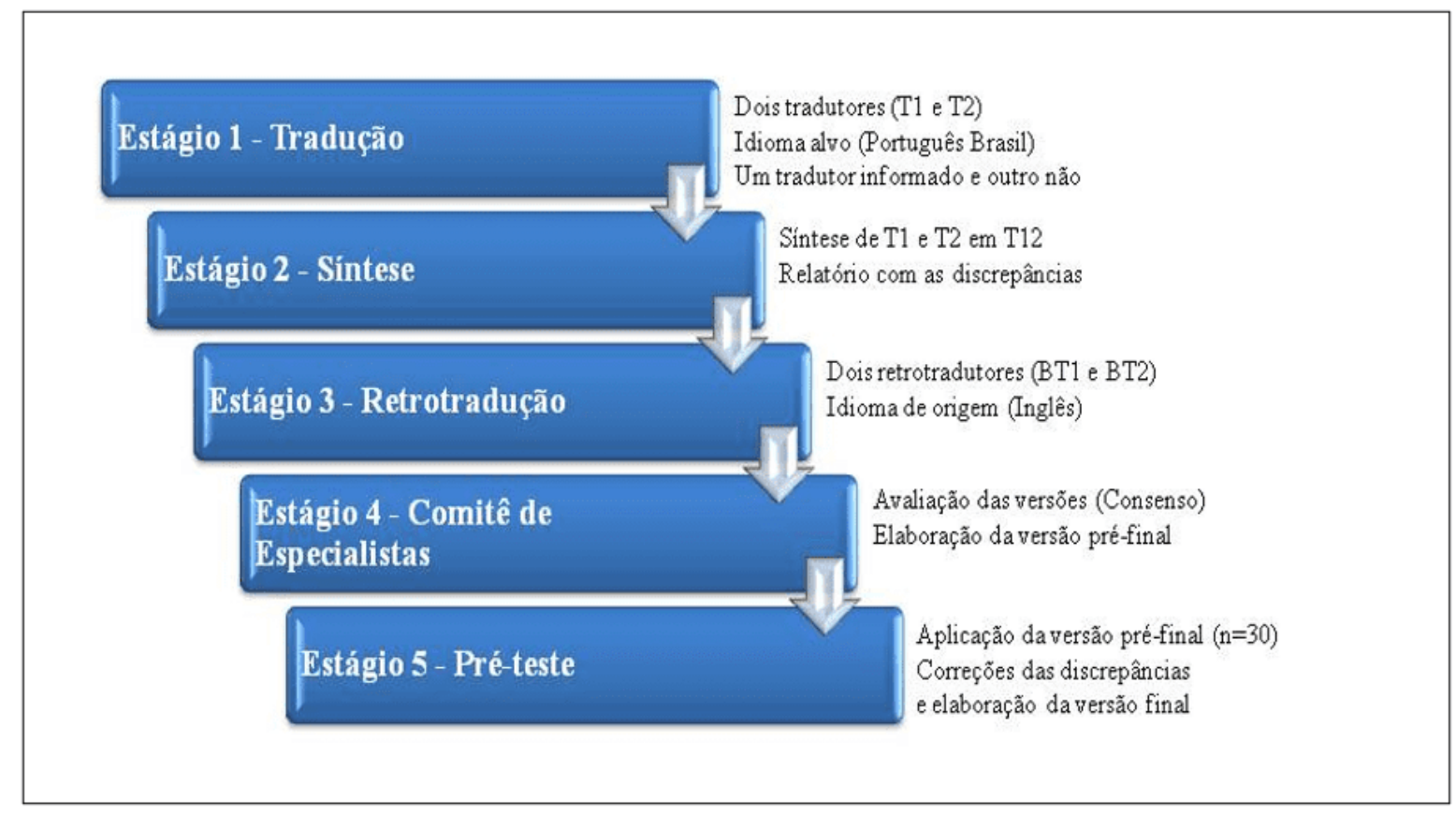

Fonte: Adaptado de Beaton et al. (2000).

A primeira etapa foi a tradução que consistiu em traduzir a JeffSATIC do inglês, para o idioma-alvo, o português, realizada dois tradutores independentes que têm o português como língua materna e fluência no inglês. As traduções foram realizadas de forma independente para obtenção de uma versão mais apropriada do material original.

Conforme referencial adotado, os tradutores possuíam diferentes perfis e informações distintas: um é profissional da área da saúde e recebera todas as 
informações sobre os objetivos do estudo e da escala, essa tradução foi denominada $\mathrm{T} 1$, e o outro tradutor sem formação na área da saúde, não recebeu qualquer informação, sendo sua tradução realizada de forma pura, literal e denominada T2. Estas traduções permitiram que o processo refletisse as características e nuanças próprias da cultura-alvo.

Na segunda etapa, denominada Síntese, uma terceira tradutora com formação na área da saúde e fluência no inglês foi responsável por comparar as traduções realizadas pelos tradutores independentes. O objetivo nessa etapa foi de reduzir discrepâncias entre o instrumento T1 e T2. Ela também foi mediadora nas discussões das diferenças entre as duas traduções. Após discussões entre a equipe, foi formulada uma síntese das duas versões traduzidas em uma única versão final traduzida, que se denominou versão-consenso - T12.

$\mathrm{Na}$ retro tradução foram realizadas duas traduções reversas independentes da versão consenso T12, feitas por dois tradutores qualificados, cuja língua de origem é o inglês e que não tinham conhecimento prévio das intenções e conceitos do material. Estas traduções foram denominadas BT1 e BT2. Ela consiste em retornar a escala em português para o inglês.

Após a conclusão dessa etapa, as versões BT1 e BT2 foram encaminhadas ao autor da escala que confirmou que ambas refletiam o mesmo conteúdo da escala original.

Finalizado o processo de tradução e tradução reversa, a versão final do instrumento foi submetida à revisão por um comitê de juízes especialistas, composto por dois profissionais doutores em distintas categorias na área da saúde com fluência no inglês, o tradutor consentido do instrumento mestre em Ciências da Saúde, um retro tradutor de origem britânica e com fluência no português, um mestre em Saúde Coletiva com experiência em tradução de instrumento, os pesquisadores e dois profissionais formados em letras com especialidade no Inglês.

Esta etapa consistiu em uma discussão com todos os membros do comitê de especialista e foi elaborada a versão brasileira da JeffSATIC. 
O comitê avaliou as seguintes equivalências:

a) Semântica: relaciona-se à equivalência gramatical e de vocabulário, pois algumas palavras de um determinado idioma podem não possuir tradução adequada para outros idiomas;

b) Idiomática: refere-se às expressões coloquiais específicas, raramente traduzíveis, sendo necessária a substituição por expressões equivalentes ou substituição dos itens;

c) Experiencial ou cultural: verifica a coerência entre os termos utilizados e as experiências vividas pela população à qual se destina;

d) Conceitual: avalia se os itens utilizados nos instrumentos têm, além de equivalência semântica, equivalência de conteúdo, capturando os mesmos conceitos.

Após essa revisão, foi elaborado um instrumento para que os membros do comitê de especialistas apontassem equivalência cultural e conceitual entre a versão brasileira e a versão original. Foi utilizada uma escala de três pontos sendo: 1 - não equivalentes; 2 - pouco equivalentes; 3 - equivalentes.

A partir da análise do Índice de Validade de Conteúdo (IVC) de quatro dos cinco membros do comitê externos à pesquisa a versão Piloto foi concluída.

A versão piloto da escala foi aprovada pelo comitê de especialistas e posteriormente aplicada como pré-teste em um pequeno número de participantes na busca de verificar a compreensão e clareza do instrumento. A amostra utilizada nessa etapa foi de 30 profissionais de diversas categorias, selecionados de forma aleatória, em unidades de Centro de Saúde da Família (CSF), do município de Goiânia. A amostra considerada ideal está entre 30 e 40 participantes. (BEATON et al., 2000).

Os participantes foram orientados a realizar sugestões que julgavam necessárias para melhorar o entendimento dos itens, bem como avaliar a diagramação do 
instrumento. Após o término do pré-teste, verificou-se que não havia necessidade de revisar qualquer item da escala, o que poderia ser definido como uma sexta etapa do processo de adaptação transcultural de acordo com Beaton et al. (2000). Após o término dessa etapa, a Escala Jefferson de Atitudes Relacionadas à Colaboração Interprofissional (EJARCI), versão brasileira da JeffSATIC foi finalizada para sua aplicação e validação.

Considerou-se população para o estudo a constituída pelos profissionais da saúde lotados em equipes de ESF vinculadas à SMS do município de Goiânia.

A população selecionada por amostragem de conveniência foi de 128 profissionais de saúde que estavam presentes nos CSF, no período de coleta de dados. A amostragem foi não probabilística, pois os dados visam aprimorar a escala por meio das avaliações de confiabilidade e não na representatividade. O tamanho da amostra deve ser de, no mínimo, cinco sujeitos por número de variáveis do instrumento, portanto superior a 100 participantes. (EPSTEIN et al., 2013).

Para o estudo, foram considerados os seguintes critérios de inclusão: Ser profissional da área Saúde da SMS de Goiânia, lotado em unidades com mais de uma equipe de Saúde da Família; aceitar participar de forma voluntária do estudo e assinar o Termo de Consentimento Livre e Esclarecido (TCLE). Como critério de exclusão: Estar lotado há menos de seis meses em uma equipe de saúde da família do município de Goiânia/GO.

Goiânia possui aproximadamente $25 \%$ da população do estado de Goiás, considerando a região metropolitana, a população é superior a dois milhões de habitantes, dados do Instituto Brasileiro de Geografia e Estatísticas (IBGE). (BRASIL, 2013).

A cobertura populacional vinculada à ESF é de aproximadamente $50 \%$, num total de 188 equipes. (BRASIL, 2015) 
A coleta de dados foi realizada no período de 15 de março a 25 de junho de 2015 , pelo próprio pesquisador.

O preenchimento da escala, durante todas as etapas do estudo, foi através de autopreenchimento dos campos pelo participante e os dados coletados foram inseridos em banco de dados específico para o processamento das análises estatísticas.

A pesquisa foi aprovada pelo Comitê de Ética em Pesquisa da Universidade Federal de Goiás (UFG)com parecer ํ․ 540.792, todos os participantes assinaram o TCLE.

A qualidade de um instrumento pode ser avaliada por meio de técnicas de validação de conteúdo, sendo elas: critério ou concorrente, de constructo e de estabilidade temporal, além de coeficientes de confiabilidade. (FACHEL e CAMEY, 2000).

Para este estudo, foi selecionada a validação de construto através do cálculo do IVC. Para a confiabilidade da escala, calculou-se o Alfa $(\alpha)$ de Cronbach. (CRONBACH, 1951)

\section{RESULTADOS}

$\mathrm{Na}$ primeira etapa do estudo, a adaptação transcultural não sofreu dificuldades expressivas ao se adaptar a linguagem. A palavra toward utilizada no título da escala foi traduzida como "relacionadas" por um tradutor e por outro como "relativa". Sendo assim, na síntese, optou-se pela tradução como sendo relacionada. $\mathrm{Na}$ síntese, também, foram acrescidos artigos definidos nos inícios das frases para padronizar o formato do questionário. Na retro tradução não foi encontrado nenhum obstáculo.

A escala é precedida por uma breve introdução em que solicita a identificação do gênero, a idade e a categoria profissional de quem responde ao instrumento. Para a formação profissional em saúde, das dezessete opções listadas na escala original, foram mantidas quatorze regulamentadas no Brasil, além do campo outros que permite especificar sua formação. (HOJAT et al., 2015).

RC: 99337

Disponível em: https://www.nucleodoconhecimento.com.br/saude/escala-jefferson 
$\mathrm{Na}$ sequência são apresentadas algumas definições para colaboração interprofissional, profissionais de saúde e paciente. Após esta primeira parte, há instruções para utilização da escala ordinal de sete pontos que deve ser utilizada para os vinte itens da escala.

Nos estágios seguintes, foram realizadas algumas modificações para que fosse atingida a equivalência semântica no desenvolvimento da versão brasileira da escala. No comitê de juízes, a definição de profissionais da saúde, as profissões "medicina e enfermagem" que apareciam distintas às demais foram suprimidas, com o intuito de não haver um sentimento de exclusão por parte dos demais profissionais que compõem equipes interprofissionais. No item 3 da escala, a tradução de enhance foi substituído o termo "intensificar" por "aumentar". No item 5, a tradução de feel foi "acharem". No item 14, a tradução de should be involved in making policy decisions ficou como sendo "devem estar envolvidos na tomada de decisões político-administrativas".

Para o item 18, a tradução de special expertise foi "competências específicas próprias", sendo esse o último item que gerou maior discussão para o melhor conceito a ser utilizado na versão brasileira.

O Quadro 1 mostra a correlação dos 20 itens da escala original em inglês e sua respectiva tradução, que é a versão adaptada culturalmente para aplicação na $A B$ no Brasil.

Quadro 1 - Adaptação transcultural dos 20 itens que compõem a Escala Jefferson de Atitudes Relacionadas à Colaboração Interprofissional.

\begin{tabular}{|l|l|l|}
\hline JeffSATIC & EJARCI \\
\hline $\begin{array}{l}\text { 1. Health professionals should be viewed as } \\
\text { collaborators rather than superiors or } \\
\text { subordinates }\end{array}$ & $\begin{array}{l}\text { deveriam ser vistos como } \\
\text { colaboradores, ao invés de } \\
\text { superiores ou subordinados. }\end{array}$ \\
\hline 2. All health professionals should have & 2. Todos os profissionais de \\
\hline
\end{tabular}


responsibility for monitoring the effects of saúde deveriam ter a interventions on their patients/clients. responsabilidade de monitorar os efeitos de intervenções em seus pacientes/clientes.

3. Teamwork in healthcare cannot be an 3. O trabalho em equipe no outcome of interdisciplinary cuidado à saúde não pode ser um education. resultado do ensino interdisciplinar.

4. Academic institutions should develop interdisciplinary educational programs to enhance collaborative practice.

4. As instituições acadêmicas devem desenvolver programas de ensino interdisciplinar para aumentar a prática colaborativa.

5. Health professionals should not question 5. Os profissionais de saúde não decisions made by colleagues even if they feel that it might have detrimental effects on the patient/client. devem questionar as decisões tomadas por colegas, mesmo que eles achem que essas possam ter efeitos prejudiciais ao paciente/cliente.

6. All health professionals can contribute to 6. Todos os profissionais de decisions regarding the well-being of saúde podem contribuir para as patients/clients. decisões relacionadas ao bemestar de pacientes/clientes.

7. Collaborative practice always works best 7. A prática colaborativa sempre when health professionals develop working funciona melhor quando os relationships to achieve agreed upon goals. profissionais de saúde desenvolvem relações de trabalho para atingir os mesmos objetivos.

8. Interdisciplinary education and 8. O ensino interdisciplinar e a interprofessional collaboration are not colaboração interprofissional não linked to one another. estão ligados entre si. 
9. The primary function of other health 9. A função principal de outros professionals is to follow, without question, profissionais de saúde é seguir, orders by the physician who are treating the sem questionamento, as patients/clients. orientações dos médicos que estão tratando

OS pacientes/clientes.

10. Interprofessional collaboration which 10

A

colaboração includes mutual respect and communication interprofissional, que inclui improves the work environment. respeito mútuo e comunicação, melhora o ambiente de trabalho.

11. All health professionals should 11. Todos os profissionais de contribute to decisions regarding improving saúde devem contribuir para as care of their patients/clients. decisões relativas à melhora do cuidado de seus

JeffSATIC pacientes/clientes.

1. Health professionals should be viewed as 1. Os profissionais de saúde collaborators rather than superiors or subordinates

\section{EJARCI} ser vistos como colaboradores, ao invés de superiores ou subordinados.

2. All health professionals should have 2. Todos os profissionais de responsibility for monitoring the effects of saúde deveriam ter a interventions on their patients/clients. responsabilidade de monitorar os efeitos de intervenções em seus pacientes/clientes.

3. Teamwork in healthcare cannot be an 3. $O$ trabalho em equipe no outcome of interdisciplinary cuidado à saúde não pode ser um education. resultado do ensino interdisciplinar.

4. Academic institutions should develop 4. As instituições acadêmicas interdisciplinary educational programs to devem desenvolver programas de 
enhance collaborative practice.

5. Health professionals should not question decisions made by colleagues even if they feel that it might have detrimental effects on the patient/client. ensino interdisciplinar para aumentar a prática colaborativa.

5. Os profissionais de saúde não devem questionar as decisões tomadas por colegas, mesmo que eles achem que essas possam ter efeitos prejudiciais ao paciente/cliente.

6. All health professionals can contribute to 6 . Todos os profissionais de decisions regarding the well-being of saúde podem contribuir para as patients/clients.

7. Collaborative practice always works best when health professionals develop working relationships to achieve agreed upon goals. decisões relacionadas ao bemestar de pacientes/clientes.

7. A prática colaborativa sempre funciona melhor quando os profissionais de saúde desenvolvem relações de trabalho para atingir os mesmos objetivos.

8. Interdisciplinary education and 8. O ensino interdisciplinar e a interprofessional collaboration are not colaboração interprofissional não linked to one another. estão ligados entre si.

9. The primary function of other health 9. A função principal de outros professionals is to follow, without question, orders by the physician who are treating the patients/clients. profissionais de saúde é seguir, sem questionamento, as orientações dos médicos que estão tratando pacientes/clientes.

10. Interprofessional collaboration which 10. colaboração includes mutual respect and communication interprofissional, que inclui improves the work environment. respeito mútuo e comunicação, melhora o ambiente de trabalho. 
11. All health professionals should 11. Todos os profissionais de contribute to decisions regarding improving saúde devem contribuir para as care of their patients/clients. decisões relativas à melhora do cuidado pacientes/clientes.

12. Job satisfaction has nothing to do with interprofessional collaborative practices.

13. Health professionals should be made aware that their colleagues in other healthrelated disciplines can contribute to the quality of care.
12. A satisfação no trabalho não está relacionada às práticas de colaboração interprofissional.

13. Os profissionais de saúde devem estar cientes de que seus colegas de outras áreas relacionadas à saúde podem contribuir para a qualidade do cuidado.
14. Health professionals should be involved in making policy decisions concerning their work.

15. Because of role differentiation, there are not many overlapping areas of responsibility among health professionals in providing care to their patients/clients.

14. Os profissionais de saúde devem estar envolvidos na tomada de decisões políticoadministrativas relativas ao seu trabalho.

15. Devido à diferença de cada função, não há muitas áreas que permitam a sobreposição de responsabilidades entre os profissionais de saúde que prestam cuidados aos pacientes/clientes.

16. To promote the best interest of the 16. Para promover 0 melhor patient/client, health professionals should benefício ao paciente/cliente, os use their own judgment rather than profissionais de saúde devem consulting their colleagues in other health- usar seu próprio julgamento ao related disciplines. invés de consultar seus colegas 
de outras áreas relacionadas à saúde.

17. Medical errors will be minimized when 17. Os erros clínicos serão collaboration exists among health minimizados quando existir a professionals. colaboração entre os profissionais de saúde.

18. All health professionals have their own 18. Todos os profissionais de special expertise to render quality care to saúde possuem competências their patients/clients. específicas próprias para prestar atendimento de qualidade aos seus pacientes / clientes.

19. Health professionals working together cannot be equally accountable for the care/service they provide.
19. Os profissionais de saúde que trabalham em conjunto não podem ser igualmente responsabilizados pelo serviço que prestam.

20. During their education, all health profession students should have experience working in teams with other health profession students in order to understand their respective role.

12. Job satisfaction has nothing to do with interprofessional collaborative practices.

13. Health professionals should be made aware that their colleagues in other healthrelated disciplines can contribute to the quality of care.
20. Durante sua formação, todos os estudantes da área da saúde devem ter a experiência de trabalhar em equipes com estudantes de outras áreas da saúde, para que possam compreender melhor sua respectiva função.

12. A satisfação no trabalho não está relacionada às práticas de colaboração interprofissional.

13. Os profissionais de saúde devem estar cientes de que seus colegas de outras áreas relacionadas à saúde podem 
contribuir para a qualidade do cuidado.

14. Health professionals should be involved in making policy decisions concerning their work.

15. Because of role differentiation, there are not many overlapping areas of responsibility among health professionals in providing care to their patients/clients.
14. Os profissionais de saúde devem estar envolvidos na tomada de decisões políticoadministrativas relativas ao seu trabalho.

15. Devido à diferença de cada função, não há muitas áreas que permitam a sobreposição de responsabilidades entre os profissionais de saúde que prestam cuidados aos pacientes/clientes.

16. To promote the best interest of the 16. Para promover o melhor patient/client, health professionals should use their own judgment rather than consulting their colleagues in other healthrelated disciplines.

benefício ao paciente/cliente, os profissionais de saúde devem usar seu próprio julgamento ao invés de consultar seus colegas de outras áreas relacionadas à saúde.

17. Medical errors will be minimized when 17. Os erros clínicos serão collaboration exists among health minimizados quando existir a professionals. colaboração entre os profissionais de saúde.

18. All health professionals have their own special expertise to render quality care to their patients/clients.

18. Todos os profissionais de saúde possuem competências específicas próprias para prestar atendimento de qualidade aos seus pacientes / clientes.

19. Health professionals working together 19. Os profissionais de saúde que 
cannot be equally accountable for the trabalham em conjunto não care/service they provide. podem ser igualmente responsabilizados pelo serviço que prestam.

20. During their education, all health 20. Durante sua formação, todos profession students should have experience os estudantes da área da saúde working in teams with other health devem ter a experiência de profession students in order to understand trabalhar em equipes com their respective role. estudantes de outras áreas da saúde, para que possam compreender melhor sua respectiva função.

Fonte: autores.

Para a validação de conteúdo foi realizada a análise por cinco juízes, especialistas, para avaliação da equivalência cultural e conceitual da escala, no entanto, somente quatro preencheram o instrumento criado para a realização das análises de cada item da escala e dessa como um todo. A partir das respostas utilizando a escala Likert de três pontos, foi calculado para a escala o IVC $=0,99$.

Após a aplicação da versão preliminar do instrumento, em uma amostra de 30 profissionais, não foi verificada a necessidade de reformular qualquer item em relação à gramática. Apenas uma sugestão em relação ao formato do questionário foi sugerida e acatada a colocação de espaço entre parênteses para a anotação do número correspondente da escala de concordância na frente de cada item, sendo essa, uma diagramação mais comum e utilizada no Brasil.

A Confiabilidade interna da escala foi obtida após análise das respostas obtidas nas 128 escalas aplicadas, com valor do Alfa de Cronbach de 0,71. 


\section{DISCUSSÃO}

A primeira etapa do estudo consistiu em realizar a adaptação transcultural da escala de atitudes e não apresentou dificuldades expressivas ao se adaptar a linguagem. $O$ termo "adaptação transcultural" é usado para abranger um processo que analisa tanto a linguagem (translação) quanto a adaptação cultural dos itens numa preparação do questionário para uso em outro ambiente. Adaptações transculturais devem ser consideradas para vários cenários diferentes segundo Beaton et al. (2000). Este trabalho assim como os estudos realizados por Correa et al. (2015) e Junkes et al. (2015) também relataram que para obtenção da equivalência semântica uma maior discussão entre os membros do comitê de especialistas fora observada, isso ocorre devido à tentativa de utilizar os melhores termos sem que haja prejuízo da equivalência conceitual.

O processo de adaptação ao qual a escala original foi submetida permitiu verificar que em sua aplicação piloto não houve necessidade de alteração de quaisquer dos itens da escala. Estudo similar realizado, também não relatou adversidades durante a aplicação da escala piloto que justificasse alteração na tradução e nova aplicação teste. A escolha dos membros para o comitê e suas respectivas especialidades $\mathrm{e}$ formações podem ter contribuído para êxito em todo o processo. (JUNKES et al., 2015)

As retro traduções foram avaliadas pelo autor da escala que confirmou que ambas continham o mesmo conteúdo da original.

O processo de tradução do instrumento de avaliação de qualidade de vida da OMS (WHOQOL-100): características e perspectivas, realizado por Fleck, foi desenvolvido através das etapas de tradução, retro tradução e discussão em grupos focais da versão com pacientes, profissionais de saúde e membros da comunidade, demonstrando que apesar das etapas seguirem o mesmo referencial adotado por este estudo há possibilidades de diferentes configurações para o comitê de especialistas. (FLECK, 2000). 
Ainda no processo de adaptação da primeira parte da escala, foram mantidas as profissões consideradas da área da saúde no Brasil conforme definidos pelo Departamento de atenção Básica. E excluídas, na versão brasileira, profissões como parteira do inglês Midwifery; médico assistente, tradução de Physician assistant, que não é uma profissão regulamentada no Brasil como é desenvolvida nos EUA; Podologia, na escala original Podiatry, que é uma profissão supervisionada e indicada por médico nos EUA. Por fim, considerando a proposta deste trabalho de aplicar e validar a escala adaptada na $A B$ em equipes ESF, também foram incluídas na relação de profissões a de Técnico e/ou Auxiliar em Saúde Bucal, no intuito de facilitar a identificação da categoria que faz parte da ESF. A alteração desses itens não interfere nos itens adaptados anteriormente e não compromete a utilização da escala. (BRASIL, 2006)

$\mathrm{Na}$ análise dos 128 instrumentos preenchidos, verificou-se que a caracterização da amostra evidencia um grande desequilíbrio entre gêneros, havendo uma proporção 5:1 de mulheres para homens. Do total de escalas, apenas quatro não tinham o campo idade respondido pelo profissional. Assim como Pinto et al. também obtiveram em seu estudo um maior número de profissionais do gênero feminino inseridos na ESF. Essa desigualdade pode não interferir na avaliação das atitudes colaborativas observadas em uma equipe interprofissional, mas sim a atuação de cada profissional dentro da equipe em que é integrante. (PINTO; MENEZES e VILLA, 2010).

Do total de 55 (42,97\%) profissionais com nível superior em Enfermagem, Medicina ou Odontologia, 49 (38,28\%) declararam possuir alguma especialização. Entre esses profissionais com especialidade, 31 (24,21\%) possuíam pós-graduação específica em Saúde Pública, Saúde Coletiva ou em Saúde da Família. Segundo Macinko; Harris e D. Phil (2015), na $A B$ do Brasil, o número de profissionais especialistas é superior ao de generalistas em uma proporção aproximada de 1,2:1, valores compatíveis do observado neste estudo. Verificar uma porcentagem elevada de profissionais de nível superior com formação específica para o nível em que 
atuam, pode garantir equipes com maior prática colaborativa e maior colaboração interprofissional.

A validade de construto foi obtida com IVC de 0,99 que é o resultado do grupo de especialistas que avaliou o processo de tradução quanto aos critérios específicos. $\mathrm{O}$ IVC consiste na avaliação do instrumento por especialistas realizado durante o processo de adaptação cultural. Ele mede a proporção da concordância dos juízes sobre determinados aspectos do instrumento e de seus itens. Inicialmente, analisa os itens da escala individualmente e posteriormente, o instrumento como um todo. (ALEXANDRE e COLUCI, 2011).

A validade de conteúdo não pode ser determinada por medidas estatísticas por se tratar do resultado do julgamento de juízes ou pessoas reconhecidas na área do estudo, que analisam a representatividade dos itens em relação aos conceitos que se pretende medir. (HILL e HILL, 1998).

Alexandre e Coluci (2011, p. 3065 apud POLIT e BECK, 2006) determinam que o quantitativo de juízes deve ser considerado para avaliação do índice que deve ser superior a 0,90 quando a avaliação for feita com menos de cinco sujeitos. Para novos instrumentos, é aceitável um índice superior a 0,80. Para as demais situações, valores abaixo de 0,78 não são recomendados. Valera et al. (2014) em estudo para verificar a equivalência de conteúdo de uma escala, afirmam que IVC obtido superior a 0,75 demonstra que $O$ instrumento atingiu sua equivalência de conteúdo. Portanto a EJARCI (IVC $=0,99)$ possui equivalência de conteúdo com a JeffSATIC e permite a sua aplicação no Brasil.

A confiabilidade da escala pode ser verificada através do coeficiente Alfa de Cronbach, que é capaz de estimar a confiabilidade de um questionário aplicado em uma pesquisa. Ele mede a correlação entre respostas em um questionário através da análise das respostas dadas pelos respondentes, apresentando uma correlação média entre as perguntas. O Alfa é calculado a partir da variância dos itens individuais e da variância da soma dos itens de cada avaliador de todos os itens de 
um questionário que utilizem a mesma escala de medição. (BITTENCOURT et al., 2011).

O coeficiente Alfa é considerado um dos métodos mais utilizados para estimar a confiabilidade interna de um instrumento, segundo Hill e Hill (1998), e seu valor é calculado considerando o número de itens que as escalas contêm, através da expressão proposta em 1951 por Lee J. Cronbach.

Com relação aos itens 3, 5, 8, 9, 12, 15, 16 e 19 que possuem $X^{-}<4$, que em uma escala de concordância de sete níveis determina a negatividade, portanto esses itens são cotados de forma inversa na escala. Isso significa que o item está em desencontro com outros itens da escala. Para que esses dados possam ser utilizados no cálculo do Alfa de Cronbach, sem que ocorra erro de codificação (a negativo) é necessária que seja feita a recodificação desses itens, ou seja, a inversão dos pontos de forma equivalente. (MAROCO e GARCIA-MARQUES, 2006).

O valor do Alfa de Cronbach calculado para a escala foi de 0,71 . De modo geral, Hill e Hill (1998) define que um instrumento ou teste é classificado como tendo confiabilidade apropriada quando o Alfa é superior a 0,70. Durante o desenvolvimento da validação de um instrumento para avaliação de disciplinas na educação superior, Bittencourt et al. (2011) obtiveram valores de alfa superiores a 0,87 para cada um dos três fatores analisados conjuntamente na escala, os quais confirmam a consistência interna do instrumento. Durante o processo de validação da escala original JeffSATIC, Hotaj et al. (2015) obtiveram valores de Alfa para a escala de 0,84 em uma amostra com 1.976 estudantes e/ou profissionais na área da saúde, em três universidades. Para a EJARCI é necessário que seja realizado estudos psicométricos com amostras representativas para verificar o comportamento da escala e sua aplicação em outros cenários.

É importante enfatizar que quanto maior for o valor do coeficiente Alfa, maior será a confiabilidade da escala. O valor de Alfa tende a aumentar com um maior número de itens no questionário, bem como quando as correlações entre os itens são mais elevadas. (HILL e HILL, 1998). 
A confiabilidade e validade de uma escala não são a mesma coisa. Uma escala pode ter boa confiabilidade e ter pouca validade. O certo é que se um instrumento não possui confiabilidade adequada, a medida não pode ter validade adequada. Em termos lógicos, pode-se afirmar que a existência de confiabilidade adequada é necessária, mas não suficiente, para garantir validade adequada (HILL e HILL, 1998)

O projeto de construção da versão brasileira da JeffSATIC foi planejado de forma a incorporar todos os aspectos teóricos necessários para que a adaptação transcultural e a tradução fossem totalmente fidedignas a escala original, garantindo a pesquisadores, estudiosos e gestores, um instrumento capaz de responder ao que se propõe.

Mas mesmo diante de uma etapa executada metodicamente dentro do proposto, ainda existe a limitação da necessidade de aplicação do instrumento em grande escala, o que permitirá diferentes análises deste e de seu produto. Nesta etapa a EJACI provou ser um instrumento capaz de fomentar o conhecimento sobre as relações de trabalho na atenção básica, contribuindo assim para o fortalecimento de políticas públicas em saúde.

No entanto vale destacar que a utilidade da $\mathrm{EJACl}$ não se limita a este espaço apenas, e que o instrumento pode ser aplicado em diferentes serviços de saúde em todos os níveis de atenção na rede pública ou privada. Contribuindo assim com a gestão de pessoas através do conhecimento das relações interprofissionais na saúde e por sua vez com a melhoria do atendimento à população, e da eficiência e eficácia de serviços de saúde através da melhoria das relações de trabalho.

\section{CONCLUSÃO}

A adaptação transcultural e tradução da JeffSATICfoi criteriosamente executada, resultando no instrumento homônimo Escala Jefferson de Atitudes Relacionadas à Colaboração Interprofissional (EJARCI), e se apresenta como instrumento de elevada equivalência conceitual, de itens e semântica com a original norte 
americana e sua validação da escala foi obtida com aplicação junto a profissionais da $A B$.

A EJARCI mostrou-se um instrumento rico e com potencial de aplicação nos mais diferentes cenários e níveis de atenção à saúde, o que permitirá subsidiar planejamentos para a formação e atuação profissional, contribuindo para melhoria dos serviços e das relações de trabalho através do conhecimento do estado das relações nos ambientes de trabalho e do estímulo a prática colaborativa em equipes interprofissionais.

\section{REFERÊNCIAS}

AGUILAR DA SILVA, R. H.; SCAPIN, L. T.; BATISTA, N. A. Avaliação da formação interprofissional no ensino superior em saúde: aspectos da colaboração e do trabalho em equipe, Campinas; Sorocaba, p. 167-184, mar. 2011. Disponivel em: <http://www.scielo.br/pdf/aval/v16n1/v16n1a09>. Acesso em: 16 fev. 2015.

ALEXANDRE, N. M. C.; COLUCI , M. Z. O. Validade de conteúdo nos processos de construção e adaptação de instrumentos de medidas. Ciência \& Saúde Coletiva, 16, n. 7, 2011.

ALONSO, L. Gestão compartilhada de conhecimento: articulação entre saberes, competências e interesses. Momento do professor. Revista de educação continuada, São Paulo, v. 2, n. 2, p. p24-31, 2005.

BEATON, D. E. et al. Guidelines for the Process of Cross-Cultural Adaptation of Self-Report Measures. Spine, v. 25, n. 24, p. 3186-3191, december 2000. ISSN 0362-2436.

BITTENCOURT, H. R. et al. Desenvolvimento e validação de um instrumento para avaliação de disciplinas na educação superior. Estudos em Avaliação Educacional, São Paulo, v. 22, n. 48, p. 91-114, abril 2011. ISSN 0103-6831. 
BRASIL. Conselho Nacional de Educação. Câmara de Educação Superior. Resolução CNE/CES N. 3, de 07 de novembro de 2001. Institui as Diretrizes Curriculares Nacionais do curso de graduação em enfermagem. Diário Oficial da República Federativa da União, Brasília, 9 novembro 2001a. Seção 1, p. 37. Disponivel em: <http://portal.mec.gov.br/cne/arquivos/pdf/CES03.pdf>. Acesso em: 21 Abril 2013.

BRASIL. Conselho Nacional de Educação. Câmara de Educação Superior. Resolução CNE/CES N. 4, de 07 de novembro de 2001. Institui as Diretrizes Curriculares Nacionais do curso de graduação em medicina. Diário Oficial da República Federativa da União, Brasília, 9 novembro 2001b. Seção 1, p. 38. Disponivel em: <http://portal.mec.gov.br/cne/arquivos/pdf/CES04.pdf>. Acesso em: 21 abr. 2013.

BRASIL. Secretaria de Atenção À Saúde. Departamento de Atenção Básica. Política Nacional de Atenção Básica. In: Pactos pela Saúde. 4. ed. Brasília: Ministério da Saúde, v. 4, 2006. p. 68.

BRASIL. Cidades@. IBGE - Instituto Brasileiro de Geografia e Estatística, 2013. Disponivel em: $<$ http://cidades.ibge.gov.br/xtras/perfil.php?lang=\&codmun=520140\&search=goias|ap arecida-de-goiania>. Acesso em: 16 setembro 2013.

BRASIL. PARECER CNE/CES №:116/2014 - Diretrizes Curriculares Nacionais para o curso de Medicina. Diário Oficial da União, Brasília, Seção 1, 06 jun. 2014. Seção 1, Pág. 17.

BRASIL. Evolução do credenciamento e implantação da estratégia Saúde da Família. Ministério da Saúde / Superintendência de Atenção à Saúde / Departamento de Atenção Básica, 2015. Disponivel em: <http://dab.saude.gov.br/historico_cobertura_sf.php>. Acesso em: 16 abril 2015. 
BRASIL. Mlnistério da Saúde. Portaria no. 2.436 de 21 de setembro de 2017, Brasília, 2017. Disponivel em: <https://www.in.gov.br/materia//asset_publisher/Kujrw0TZC2Mb/content/id/19308123/do1-2017-09-22-portaria-n-2436-de-21-de-setembro-de-2017-19308031>. Acesso em: 30 out. 2019.

CÂMARA, A. M. C. S. et al. Educação interprofissional no Brasil: Cosntruindo redes formativas de educação e trabalho em saúde. Interface Comunicação Saúde Educação, v. 20, n. 56, p. 9-12, 2016.

CECCIM, R. B.; FEUERWERKER, L. C. M. Mudança na graduação das profissões de saúde sob o eixo da integralidade. Cadernos de Saúde Pública [online], 20, n. 5, 2004. 1400-1410.

CIHC - CANADIAN INTERPROFESSIONAL HEALTH COLLABORATIVE. Inventory of Quantitative Tools Measuring Interprofessional Education and Collaborative Practice Outcomes. Toronto: [s.n.], 2012. 68 p.

CORREA, L. S. et al. Tradução e adaptação transcultural do instrumento da Organização Mundial da Saúde sobre o uso de sinais de alarme para dengue por profissionais de saúde. Cadernos de Saúde Pública [online], 31, n. 2, 2015. 247-256.

COOKE, M. et al. American Medical Education 100 Years after the Flexner Report. The new england journal of medicine, Massachusetts, 28 Sep. 2006. 133944.

CRONBACH, L. J. Coefficient alpha and the internal structure of tests. Psychometrika, Illinois, 16, n. 3, sep. 1951.

DE SOUZA ARAÚJO, M. B.; DE MEDEIROS ROCHA, P. Trabalho em equipe: um desafio para a consildicação da estratégia de saúde da família. Ciência \& Saúde Coletiva [online], v. 12, n. 2, p. 455-464, abril 2007. ISSN 1413-8123. 
EPSTEIN, J. et al. Crosscultural adaptation of the Health Education Impact Questionnaire: experimental study showed expert committee, not backtranslation, added value. Journal of Clinical Epidemiology, 68, n. 4, 2013. 360-9.

FACHEL, J. M. G.; CAMEY, S. A. Avaliação Psicométrica: a qualidade das medidas e o entendimento dos dados. In: CUNHA, J. A. Psicodiagnóstico. 5. ed. Porto Alegre: Artes Médicas, 2000. Cap. 14, p. 158-170.

FLECK, M. P. D. A. O instrumento de avaliação de qualidade de vida da Organização Mundial da Saúde (WHOQOL-100): características e perspectivas. Ciência \& Saúde Coletiva, 5, n. 1, 2000. 33-38.

FORTUNA, C. M. et al. O trabalho de equipe no programa de saúde da família: reflexões a partir de conceitos do processo grupal e de grupos operativos. Revista Latino-Americana de Enfermagem, Ribeirão Preto, 13, n. 2, mar./abr. 2005.

HILL, M. M.; HILL, A. Investigação empírica em ciências sociais: um guia introdutório. 2ª ed. [S.I.]: Dinâmica, 1998.

HOJAT, M. et al. Comparisons of American, Israeli, Italian and Mexican physicians and nurses on the total and factor scores of the Jefferson scale of attitudes toward physician-nurse collaborative relationships. International Journal of Nursing Studies, v. 40, n. 4, p. 427-435, may 2002. ISSN 0020-7489.

HOJAT, M. et al. The Jefferson Scale of Attitudes Toward Interprofessional Collaboration (JeffSATIC): development and multi-institution psychometric data. Journal of Interprofessional Care, 29, n. 3, may 2015. 238-44.

HOJAT, M.; HERMAN, M. W. Developing and instrument to measure attitudes toward nurses: preliminary psychometric findings. Psychological Reports, 56, 1985. 571-579. 
IRIBARRY, I. Aproximações sobre a transdisciplinaridade: algumas linhas históricas, fundamentos e princípios aplicados ao trabalho de equipe. Psicologia: Reflexão e Crítica, 16, n. 3, 2003. p.483-490.

JUNKES, M. C. et al. Validity and Reliability of the Brazilian Version of the Rapid Estimate of Adult Literacy in Dentistry - BREALD-30. Public Library of Science One, 9 jul. 2015.

LAMPERT, J. B. Na Transição Paradigmática da Educação Médica: 0 que o paradigma da integralidade atende que o paradigma Flexneriano deixou de lado. Cadernos da Associação Brasileira de Educação Médica, 2004. Disponivel em: $<$ http://www.abem-educmed.org.br/pdf_caderno1/ jadete_final.pdf>. Acesso em: fev. 2015.

LEMOS, L. L.; REINEIROS, ; DE MORAIS,. A Transdisciplinaridade no trabalho em equipes do programa saúde da família: um estudo de caso. Brasília: [s.n.]. 2006.

MACINKO, J.; HARRIS, M. J.; D.PHIL, B. S. Brazil's Family Health Strategy Delivering Community-Based Primary Care in a Universal Health System. The New England Journal of Medicine, 372, n. 23, 04 jun. 2015. 2177-2181.

MAROCO, ; GARCIA-MARQUES,. Qual a fiabilidade do alfa de Cronbach? Questões antigas e soluções modernas? Instituto Superior de Psicologia Aplicada, Portugal, 4, n. 1, 2006. 65-90.

OMS. Marco para Ação em Educação Interprofissional e Prática Colaborativa (WHO/HRH/HPN/10.3). Tradução de Rede de Profissões de Saúde - Enfermagem \& Obstetrícia do Departamento de Recursos Humanos para a Saúde. Genebra: Freelance, 2010.62 p.

PEDUZZI, et al. Trabalho em equipe na perspectiva da gerência de serviços de saúde: instrumentos para a construção da prática interprofissional. Physis: Revista de Saúde Coletiva, Rio de Janeiro, 21, n. 2, 2011. 
PEDUZZI, et al. Educação interprofissional: formação de profissionais de saúde para o trabalho em equipe com foco nos usuários. Revista da Escola de Enfermagem da USP, São Paulo, 47, n. 4, aug; 2013. http://dx.doi.org/10.1590/S0080-623420130000400029.

PEDUZZI, M. . N. I. J. . G. A. C. G. . S. J. A. M. . S. G. C. Educação interprofissional: formação de profissionais de saúde para o trabalho em equipe com foco nos usuários. Rev Esc Enferm USP, São Paulo, v. 4, n. 47, p. 977-83, 2015.

PEDUZZI, M.; CIAMPONE, M. H. T. Trabalho em equipe. Rio de Janeiro: Guanabara Koogan, 2005. 108-24 p.

PEDUZZI, M.; CIAMPONE, M. H. T. Trabalho em equipe e processo grupal. In: KURCGANT, P. Gerenciamento em enfermagem. Rio de Janeiro: Guanabara Koogan, 2005. p. 108-24.

PINTO, E. S. G.; MENEZES, R. M. P. D.; VILLA, T. C. S. Situação de trabalho dos profissionais da Estratégia Saúde da Família em Ceará-Mirim. Revista da Escola da Enfermagem da USP, 44, n. 3, set. 2010. 657-664.

SOMMERMAN, A. Inter ou transdisciplinaridade? da fragmentação disciplinar ao novo diálogo entre os saberes. São Paulo: Paulus, 2006. 75 p.

SOUZA, G. C. D. et al. Trabalho em equipe de enfermagem: circunscrito a profissão ou colaboração interprofissional. Rev Esc Enferm USP, São Paulo, v. 50, n. 4, p. 642-649, 2016.

TRAD, L. A. B. 0 trabalho em equipe no PSF: investigando a articulação técnica e a interação entre os profissionais. Interface (Botucatu) [online], v. 9, n. 16, p. 2538, fevereiro 2005. ISSN 1807-5762. 
VALERA, G. G. et al. CULTURAL ADAPTATION OF THE SCALE PAIN ASSESSMENT IN ADVANCED DEMENTIA - PAINAD TO BRAZIL. Revista da Escola de Enfermagem da USP, Ribeirão Preto, 48(3), n. 3, 2014. 462-8.

VANDERBILT, A. A.; DAIL, M. D.; JABERI, P. Reducing health disparities in underserved communities via interprofessional collaboration across health care professions. Journal of Multidisciplinary Healthcare, 8, 21 apr. 2015. 205-208.

WHO. Framework for Action on Interprofessional Education \& Collaborative Practice (WHO/HRH/HPN/10.3). Geneva: World Health Organization, Department of Human Resources for Health, 2010.

WIECHA, J.; POLLARD, T. The interdisciplinary e health team: chronic care for the future. Journal of Medical Internet Reserch, Toronto, 09 mar. 2004. Vol.6, e22.

Enviado: Outubro, 2021.

Aprovado: Outubro, 2021. 